\title{
Ino Arndt
}

\section{Luxemburg}

\section{Deutsche Besetzung und Ausgrenzung der Juden}

Zu Beginn des Westfeldzuges, am frühen Morgen des 10. Mai 1940, marschierten deutsche Truppen - unerwartet für die Alliierten - in Luxemburg ein und verletzten damit den Neutralitātsstatus des Landes. Der um mehrere Stunden verspātete franzōsische Gegenangriff im Süden des Landes konnte den Vormarsch der 16. Armee nicht aufhalten, so daß bis zum 13. Mai die deutschen Verbände die Westgrenze des Großherzogtums überschritten hatten. ${ }^{1}$ Aus Protest gegen den Neutralitātsbruch ging die Landesfürstin Charlotte noch am 10. Mai 1940 mit dem Kabinett ins Exil. Eine von der Abgeordnetenkammer eingesetzte Regierungskommission unter Leitung von Generalsekretār Albert Wehrer amtierte als Exekutive. Entgegen der vom deutschen Gesandten, Otto von Radowitz, abgegebenen Erklärung, seine Regierung werde die territoriale und politische Integrität Luxemburgs nicht antasten ${ }^{2}$, entschied Ribbentrop am 16. Mai, das Großherzogtum als feindliches Land zu betrachten. Damit handelte et auch gegen den Wunsch von OKW und OKH vom 13. Mai, es möge deutscherseits davon Abstand genommen werden, sich als mit Luxemburg im Kriegszustand befindlich zu betrachten ${ }^{3}$, und schließlich gegen den Vorschlag der Politischen Abteilung des Auswärtigen Amtes, „bis auf weiteres“ so, d.h. entsprechend dem Wunsche der Militärs zu verfahren. ${ }^{4}$ Darüber hinaus akzeptierte Ribbentrop Wehrer nur als Vertreter der Landesbehörden, nicht jedoch als Repräsentant der luxemburgischen Regierung5; am 21. Mai wurde der deutsche Gesandte Otto von Radowitz abberufen. 6

Die Exekutivgewalt ging nun an die Militärverwaltung über, d.h. an den Militärbefehlshaber von Belgien und Nordfrankreich, General von Falkenhausen, bzw. seinen Vertreter in Luxemburg, Oberst Schumacher, dem Chef der Feldkommandantur $515 .^{7}$ Die Militārverwaltungszeit endete schon nach knapp drei Monaten, als durch Erlaß Hitlers vom 2. August 1940 einer der, alten Kämpfer“, der Gauleiter von Koblenz-

1 Vgl. E. T. Melchers, Kriegsschauplatz Luxemburg August 1914, Mai 1940, Luxemburg 19794, S. 467-472.

2 ADAP, Serie D, Bd. IX, S. 249.

3 Ebenda, S. 273.

4 Ebenda, S. $281 \mathrm{f}$.

5 Ebenda, S. 290.

6 Ebend2, S. 330.

7 Gemäß Hitlers Erlaß über die Verwaltung der besetzten Gebiete Frankreichs, Luxemburgs, Belgiens und Hollands vom 9.5.1940 hatte die Handhabung der Militarverwaltung so zu erfolgen, daß der "Eindruck einer beabsichtigten Annektion der besetzten Gebiete nicht entsteht“, IfZ, MA-190/1, Bl. 1720424 f. 
Trier, Gustav Simon, zum Chef der Zivilverwaltung (CdZ) ernannt wurde. ${ }^{8}$ Als sein Vertreter fungierte der Regierungspräsident von Trier, Heinnich Siekemeier. Leiter der Gestapo in Luxemburg war seit dem 11. Mai SS-Sturmbannführer Wilhelm Nölle, der auch die Führung des am 16. August errichteten Einsatzkommandos Luxemburg übernahm; Anfang März 1941 wurde er von SS-Obersturmbannführer Dr. Fritz Hartmann abgelöst.9

Einen Monat nach seinem Amtsantritt begann Simon damit, die im Großherzogtum lebenden Juden - einheimische und ausländische, die zum großen Teil aus Deutschland geflüchtet waren - den schon im Reichsgebiet geltenden antijüdischen Gesetzen zu unterwerfen. Am 5. September trat die „Verordnung über Maßnahmen auf dem Gebiet des Judenrechts ${ }^{\star 10}$ in Kraft; sie übertrug die unter dem Begriff „Nürnberger Gesetze" bekannt gewordenen Diskriminierungen auf Luxemburg. Vom selben Tag datiert die "Verordnung über das jüdische Vermōgen in Luxemburg ${ }^{411}$, die die Juden dazu verpflichtete, ihr gesamtes in- und ausländisches Eigentum anzumelden (am 18. Dezember wurde eine Fristverlängerung bis Jahresende 1940 gewāhrt $^{12}$ ) und ihnen untersagte, ohne Genehmigung gewerblichen, land- und forstwirtschaftlichen Besitz zu veräußern, zu verpachten oder zu erwerben; analoges galt für Edelmetalle, Schmuck und Kunstwerke. Betroffen von diesen vermögensrechtlichen Bestimmungen waren Juden luxemburgischer und deutscher Staatsangehōrigkeit.

Das Berufsverbot ${ }^{13}$ für jüdische Ärte, Tierärzte, Zahnärzte, Apotheker und Rechtsanwālte und die Anordnung, daß diejenigen Juden, die Träger eines öffentlichen Amtes - im weitesten Sinne des Wortes - waren, ihre Tätigkeit sofort einzustellen hatten ${ }^{14}$, fanden aus unbekannten Gründen keinen Niederschlag im offiziellen Verordnungsblatt der Zivilverwaltung. Sie sind wohl zur gleichen Zeit mit den obengenannten Verordnungen als "unverzüglich“ durchzuführende Weisungen Simons an die inzwischen auch nominell zur Verwaltungskommission herabgestufte Regierungskommission ${ }^{15}$ ergangen.

8 Nbg. Dok. NG-4297, NOKW-3474 (s.a. IfZ, MA-444/3, Bl. 3964 f.). Praktisch hat Simon schon am 29.7.1940 sein Amt angetreten; vgl. Willard Allen Fletcher, The German Administration in Luxemburg 1940-1942, in: The Historical Journal 13 (1970), S. 533-544.

9 IfZ, MA-444/3, Bl. 3983 und MA-436, Bl. 945 ff. Nölle ist wegen Differenzen mit Simon in der Frage der Judenausweisungen schon Ende Februar von Hartmann abgelöst worden. Beide waren im Luxemburger Gestapo-Prozeß angeklagt, das Urteil vom Februar 1951 sah für Nölle (in Abwesenheit) 12 Jahre Zwangsarbeit vor, Hartmann wurde zum Tode verurteilt, später begnadigt und Ende 1957 in die Bundesrepublik abgeschoben; vgl. BA, All. Proz. 21/261 und 282 und Paul Cerf, Longtemps j’aurai mémoire. Documents et témoignages sur les juifs du Grand-Duché de Luxembourg durant la seconde guerre mondiale, Luxembourg 1974, S.134 ff. Dieses gut dokumentierte, seit langem vergriffene Buch galt zu Recht als Standardwerk über die Judenverfolgung in Luxemburg, bis Cerf 1986 eine überarbeitete und ergänzte Fassung unter dem Titel L'Etoile Juive au Luxembourg, Luxembourg 1986, herausbrachte.

10 Vgl. Verordnungsblatt für Luxemburg, hrsg. vom Chef der Zivilverwaltung in Luxemburg (im folgenden zitiert VOBI) 1940, S. 10 f.; entsprechende reichsrechtliche Durchführungsvorschriften wurden in Luxemburg durch die VO vom 31.1.1941 eingeführt (vgl. VOBI, S. 118).

11 VOB] 1940, S. $11 \mathrm{ff}$.

12 VOBl 1940, S. 433.

13 Vgl. dazu „Die Judenfrage“, hrsg. von der „Antisemitischen Aktion“ (Nachfolgerin des 1934 vom Propagandaministerium errichteten „Instituts zur Erforschung der Judenfrage“, vgl. Helmut Heiber, Walter Frank und sein Reichsinstitut für die Geschichte des neuen Deutschlands, Stuttgart 1966, S. 602), vom 15.10.1940, S. $156 \mathrm{ff}$.

14 Nbg. Dok. NG-2297.

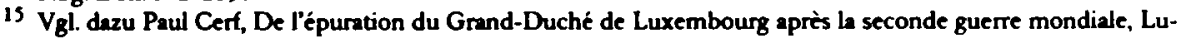
xembourg 1980, S. 94. 
Der Einführung dieser ersten Restriktionen war Ende August 1940 ein Schreiben des $\mathrm{CdZ}$ an das Reichsinnenministerium vorausgegangen, in dem Simon die Entwürfe für die drei Maßnahmen dem dafür zustāndigen Reichsminister Frick ${ }^{16}$ mit der Bitte um Zustimmung vorgelegt hatte. Der Reichsinnenminister seinerseits bat am 31. August - unter Berufung auf die Entscheidung Hitlers, deutsches Recht in Luxemburg nur begrenzt einzuführen - die Reichskanzlei, gegebenenfalls darüber eine neuerliche Entscheidung des Führers herbeizuführen. Dessen bedurfte es jedoch nicht mehr, da sich Simon in der Zwischenzeit schon persōnlich und zweifellos mit dem Hinweis auf seine, in der vom 2. August verfügten unmittelbaren Unterstellung unter Hitler an diesen gewandt und von ihm die Zustimmung zu den geplanten Maßnahmen erhalten hatte. 17

Seit Ende 1940/Anfang 1941 wurden in rascher Folge - so, als wollte man das im Reichsgebiet innerhalb von acht Jahren geleistete Pensum antisemitischer Gesetzgebung mōglichst schnell nachholen - zahlreiche weitere Bestimmungen erlassen ${ }^{18}$, die die Juden mehr und mehr diskriminierten und ihr tägliches Leben in zunehmendem Maße beeinträchtigten. Teils kamen diese Bestimmungen von der Zivilverwaltung auf dem amtlichen Verordnungsweg, teils als Bekanntmachungen des Konsistoriums der Israelitischen Kultusgemeinde bzw. des seit 14. April 1942 von den Deutschen eingeführten Ältestenrates (conseil juif aînés) ${ }^{19}$, die ihrerseits auf Anordnungen (meist) der Gestapo, der Sicherheitspolizei oder auch der Zivilverwaltung tätig werden mußten. Anfang Dezember 1940 erging das Schāchtverbot, drei Wochen spāter wurde verfügt, daß Rechtsgeschāfte, an denen ein Jude (oder ein jüdischer Gewerbebetrieb) beteiligt war, zur Rechtswirksamkeit der Genehmigung des $\mathrm{CdZ}$ bedurften. Kinderreiche jüdische Familien erhielten weder Ausbildungs- noch Kinderbeihilfen, außerdem wurden auch keine Ehestandsdarlehen mehr gewährt. Ab Januar 1941 mußten die Juden eine Sozialausgleichsabgabe in Höhe von 15 Prozent ihres Lohnes oder ihrer sonstigen Bezüge leisten. Anfang Februar wurde bestimmt, daß das Vermögen geflohener und emigrierter Juden - rückwirkend zum 10. Mai 1940 - deutscher Verwaltung unterlag und vom CdZ eingezogen werden konnte; gleiches galt ab Mitte April für das Vermögen der noch in Luxemburg lebenden Juden und seit Anfang Juli folgerichtig auch für das von verstorbenen Juden.

Mit der Durchführung der Bestimmungen über das Vermōgen von Juden war im Herbst 1940 der bereits im Gau Koblenz-Trier mit der Arisierung betraute Gauinspektor Josef Ackermann beauftragt worden. Die Ende Mai 1941 veröffentlichte Bilanz seiner dreivierteljāhrigen Arbeit ${ }^{20}$ : von 335 jüdischen Betrieben waren 75 ,arisiert" und mit kommissarischen Verwaltern besetzt, die übrigen „beseitigt"; er hatte außerdem 380 bebaute Grundstücke und 155 ha unbebauten Grundes ermittelt.

16 Mit dem Erlaß vom 2.8.1940 (vgl. Anm. 8) war der Reichsminister des Innern als Zentralstelle für Luxemburg" (analog für Elsaß und Lothringen) mit der Aufgabe, für eine einheitliche, auf die Bedürnisse dieses Gebietes abzustimmende Zusammenarbeit der Obersten Reichsbehörden untereinander und mit dem Chef der Zivilverwaltung Sorge zu tragen“, eingesetzt worden. Vgl. dazu auch Lothar Kettenacker, Nationalsozialistische Volkstumspolitik im Elsaß, Stuttgart 1973, S. $59 \mathrm{ff}$.

17 Nbg. Dok. NG-2297.

18 Die folgenden neun Verordnungen mit entsprechenden Durchführungsverordnungen sind im VOB] 1940,

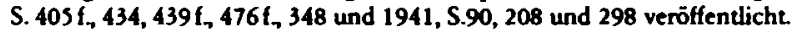

19 Paul Cert, L'Etoile juive, S. 116.

20 "Die Judenfrage" (vgl. Anm. 13), 31.5.1941, S. 97. 
Durch Zwangsausweisung ${ }^{21}$ der ehemaligen jüdischen Bewohner freigewordene Hãuser hatte man den deutschen Behörden zur Verfügung gestellt oder an in Luxemburg ansāssige Deutsche verkauft das Ackerland verpachtet oder volksdeutschen Bauern zum Kauf angeboten und das bei Flucht oder Emigration zurückgelassene Mobiliar bedürftigen Familien überlassen.

Neben der Fülle der amtlichen Maßnahmen, mit Hilfe derer die Juden in Luxemburg Schritt für Schritt ihrer Rechte und ihres Vermögens beraubt wurden, gab es eine quasi inoffizielle Verfolgung ${ }^{22}$, die deutlich macht, wie stark die als Menschen minderer Klasse eingestuften Juden der Willkür und Schikane nationalsozialistischer Funktionäre preisgegeben waren. Bei der Mehrheit der Luxemburger fand sie allerdings keine aktive Unterstützung. Doch einige kieinere antisemitische und nazistische Gruppierungen hatten schon vor dem Einmarsch der Deutschen mit anti-jüdischen Aktionen auf sich aufmerksam gemacht. Treffpunkt dieser Akteure war das "Café de la Poste", dessen Besitzer Christophe Bintner bereits 1932 sein Lokal für ,judenfrei“ erklärt hatte. Bei den antisemitischen Aktionen der folgenden Jahren 1933-1940 spielte das Café eine wichtige Rolle. ${ }^{23}$ Als die Deutschen im Mai 1940 in Luxemburg einfielen, konnten sie sich der Kollaboration dieser deutschgesinnten Gruppierungen, die sich im Juli 1940 zur „Volksdeutschen Bewegung“ (VdB) zusammenschlossen, sicher sein. Eine Beteiligung der VdB an den antisemitischen Aktionen, die Ende Oktober 1940 einsetzten, kann zwar nicht nachgewiesen werden, ist aber anzunehmen. Unbekannte brachen in die Synagoge in Ettelbrück ein, zerstörten das Mobiliar und die Kultgegenstānde; jegliche gerichtliche Untersuchung unterblieb. Jüdische Häuser in Mersch wurden aufgebrochen, Mobiliar gestohlen; nach einem Brandanschlag auf die Synagoge in Luxemburg im Februar 1941 wurde dem Israelitischen Konsistorium die Feuerversicherung gekündigt. Mitglieder des National-Sozialistischen Kraftfahr-Korps (NSKK) drangen am Freitag, dem 9. Mai 1941 während des abendlichen SchabbatGottesdienstes in dieselbe Synagoge ein, drohten mit der Sprengung des Gebäudes, knüppelten die fliehenden Gläubigen nieder und verwüsteten die Einrichtung. Als eine Woche spāter der Großrabbiner - seit 1929 Dr. Robert Serebrenik - tātlich angegriffen wurde, weigerte sich die Polizei einzuschreiten. Verschiedene Brandanschläge auf das Gotteshaus in der Hauptstadt dienten den deutschen Behörden Mitte Mai 1941 als Vorwand, Schließung und Abbruch ${ }^{24}$ der Synagoge anzuordnen.

Die zweifellos einschneidendste der von Simon seit seiner Amtsübernahme ergriffenen antijüdischen Maßnahmen war die ab Anfang August 1941 wirksame "Verordnung betr. Ordnung des jüdischen Lebens in Luxemburg “. ${ }^{25}$ Sie war die Vorstufe zur

21 Vgl. dazu Cerf, Longtemps, S. $51 \mathrm{ff}$.

22 Ebenda. Vgl. auch den anläMlich der Einweihung eines Mahnmals im KJoster Fünfbrunnen (Juli 1969) von Evy Friedrich veröffentlichten Bericht "Von Fünfbrunnen nach Auschwitz" über Verfolgung und Deportation der Juden in Luxemburg, in: Revue (Luxembourg). Nr. 22 und 23, vom 31.5 und 7.6.1969. Im Verlauf einer von luxemburgischen Anhängem der NSDAP bzw. potentiellen Mitgliedern der am 13.7.1940 gegründeten „Volksdeutschen Bewegung“ (VdB) ausgeführten KJebe-Aktion mit pro-NS-Parolen Anfang Juli 1940 waren auch zahlreiche Zettel und Plakate mit antijüdischen Parolen geklebt worden, vgl. Paul Spang, Von der Zauberfōte zum Standgericht. Naziplakate in Luxemburg, Luxemburg 1982 und Melchers, Kriegsschauplatz, S. 607.

23 Cerf, L'Etoile Juive, S. 28 f. und S. 74.

24 Für den Abbruch, der sich bis zum Herbst 1943 hinzog, fand sich keine Luxemburger Firma bereit, vgl. Cerf, Longtemps, S. 52.

25 VOBI 1941, S. 325 . 
Ghettoisierung, die sich wenig später noch verschärfen sollte: den Juden wurde der Besuch von Gaststātten, Theatem, Kinos, Sportplātzen, Badeanstalten und öffentlichen Veranstaltungen verboten, man beschränkte ihre Einkaufszeit auf zwei Stunden täglich in wenigen (schon im Mārz 1941 dafür zugelassenen ${ }^{26}$ ) Geschäften, verhāngte eine Ausgangssperre zwischen 19 und 7 Uhr und führte - früher als im Reichsgebiet die Kennzeichnungspflicht (gelbe Armbinde) ein. Ab 14. Oktober 1941 wurde die Armbinde durch einen Judenstem ersetzt. Nachgeholt wurde eine andere, in Deutschland schon drei Jahre zuvor dekretierte zweite Form der Kennzeichnung: als zusātzlichen Vornamen mußten die luxemburgischen und staatenlosen Juden seit dem 1. Oktober den Namen Israel bzw. Sara annehmen, sofem sie nicht schon einen jüdischen Vornamen aus der vom Reichsinnenministerium im August 1938 veröffentlichten Liste trugen. Namensänderungen mußten den zustāndigen Behörden schriftlich bekanntgegeben werden. ${ }^{27}$

Die Verordnung über das jüdische Leben in Luxemburg wurde bereits zweieinhalb Monate nach ihrem Inkraftreten durch eine zweite wieder aufgehoben ${ }^{28}$, die eine noch weitergehende Beschrānkung der ohnehin schon minimalen Rechte der Juden brachte und deren wesentlichste Bestimmung den administrativen Abschluß der Vorbereitungen für die erste Deportation nach Polen bedeutete: Ohne schriftliche Erlaubnis der Polizei durften die Juden ihren Wohnort nicht mehr verlassen. Femer war ihnen die Benutzung der Straßenbahn von nun an verboten.

In den folgenden Jahren blieb den Deutschen in dem seit 30. August 1942 auch de facto annektierten ${ }^{29}$ Großherzogtum nur noch wenig zu tun, um die Juden ihrer wenigen noch verbliebenen Rechte zu berauben. Verboten wurde ihnen u.a. ${ }^{30}$ jeglicher Umgang mit Nicht-Juden (unter Strafandrohung der Einweisung in ein Konzentrationslager), das Benutzen von Telefonen und der Besitz von Haustieren sowie das Abonnieren von Zeitungen und Zeitschriften. Bereits seit dem 12. Mai 1942 mußten jüdische Wohnungen gekennzeichnet werden und am 30. Juni wurde die Schließung der jüdischen Schule - einzige noch verbliebene Unterrichtsmöglichkeit für die jüdischen Kinder - angeordnet. Neben der verordneten Ablieferung (mit Liste in dreifacher Ausfertigung und Strafandrohung bei Nichtbefolgung) verschiedenster Bedarfsdinge des täglichen Lebens, blieb dem $\mathrm{CdZ}$ noch die Befugnis zu erteilen, das eingezogene Vermōgen der Juden (und Emigranten) an Dritte weiterzugeben. ${ }^{31}$ Schließlich wurde am 9. Juli 1943 die berüchtigte 13. Verordnung zum Reichsbürgergesetz eingeführt, womit die Juden auf ngesetzlichem ${ }^{4} \mathbf{W e g}$ in die völlige Rechtlosigkeit gestoßen wurden. 32

26 Cert, Longtemps, S. 55.

27 VOBI 1941, S. 407 und RGBI I, 1938, S. 1044.

28 VOBI 1941, S. 420 f. Das andere, vergleichsweise unerhebliche Verbot, Friseurgeschäfte aufzusuchen, war eine Vorwegnahme der im Reichsgebiet erst Mitte Mai 1942 wirksam gewordenen entsprechenden Maßnahme.

29 Von einem de-facto-Anschluß zu sprechen scheint begründet angesichts der beiden in Luxemburg am 30.8.1942 eingeführten Verordnungen über die Staatsangehörigkeit und die Wehrpflicht (VOBI 1942, S. 253 ff.h vgl. dazu Fletcher, German Administration, S. 544.

$30 \mathrm{Vgl}$. Cerf, Longtemps, S. $58 \mathrm{ff}$.

31 VOBI 1942, S. 134.

32 VOBI 1943, S. 154. 


\section{Auswanderung und Deportation}

Das Großherzogtum hatte - laut Volkszählung vom 31.12.1935 - 295213 Einwohner, darunter 3144 (Konfessions-)Juden ( $=1,06 \%$ der Bevōlkerung). ${ }^{33}$ Von diesen waren 870 luxemburgischer und 2274 auslāndischer Nationalitāt. Die seit der Volkszāhlung von 1930 auffallende Zunahme der Zahl ausländischer Juden von 1526 auf 2274 (d.h. um 49\%) - bei Abnahme der ausländischen Bevölkerung um 3,18\% - im Vergleich zu der der einheimischen von 716 auf 870 (d.h. um 21,5\%), ist in erster Linie auf eine große Immigration von Juden aus Deutschland und dem Saargebiet von Herbst $1933^{34}$ bis Ende 1935 zurückzuführen: innerhalb dieser Zeit stieg ihre Zahl von 463 auf 1096, das bedeutete eine Zunahme um 136,7\%. Uber die zahlenmäßige Weiterentwicklung der jüdischen Einwanderung aus Deutschland bzw. die Zahl der Immigranten aus Ósterreich und der Tschechoslowakei liegen keine statistischen Daten vor; gleiches gilt für die Entwicklung der eingesessenen jüdischen Bevölkerung.

Der Versuch, die Verluste der Juden - einheimischer wie zugewanderter - in Luxemburg exakt festzustellen, stößt auf verschiedene Schwierigkeiten. So ist nicht bekannt, wieviele Juden am 10. Mai 1940 im Großherzogtum lebten; die Schātzungen schwanken 2 wischen 3500 und $5000 .{ }^{35}$ Ungewilheit besteht ferner über die Zahl der Juden, die am 10. Mai oder unmittelbar danach entweder im Zuge einer allgemeinen Fluchtbewegung oder der Evakuierung der Südgemeinden durch die franzōsischen Truppen ${ }^{36}$ Luxemburg verlassen haben; entsprechende Schätzungen für diesen ersten Exodus der Juden liegen zwischen 800 und 2500.37 Auch nicht genau zu ermitteln ist schließlich weder die Zahl der seit dem 10. Mai 1940 emigrierten Juden ${ }^{38}$ noch jener,

$33 \mathrm{Vgl}$. Résultats du recensement de la population du 31 décembre 1935. Grand-Duché de Luxembourg - Publications de l'Office de Statistique. Fascicule 69, Luxembourg 1938. In der Konfessions-Statistik (S. 63) werden 5653 Personen genannt, die entweder keiner Konfession angehörten oder keine angegeben hatten. Wieviele von diesen $(1,9 \%$ der Bevoolkerung) Juden im Sinne der NS-Terminologie waren, ist nicht bekanne.

34 Vor 1933 sind keine statistischen Erhebungen über die Konfessionszugehörigkeit der Ausländer angestellt worden, ebenda, S. 78; hinzuweisen ist hier noch auf eine Zunahme der deutschen Immigration aus dem Saarland und dem Reichsgebiet (ohne Rheinland) zwischen 1930 und 1935 um 3 bzw 6\%; vgl. ebenda, S. 931 .

35 Vgl. Cerf, Longtemps, S. 26f.; Nicolas Bosseler/Raymond Steichen (Hrsg.th Livre d'Or de la Résistance Luxembourgoise de 1940-1945, Esch-sur-Azette 1952, S. 569; Encyclopaedia Judaica, vol. XI, S. S91; Hitler's Ten Year War on the Jews, hrsg. vom Institute of Jewish Affairs, New York 1943, S. 257.

Cerf geht in seinem neuesten Buch, L'Etoile Juive (S. 21 und 177), von 3700 aus, wovon etwa 1600 Einwanderer waren.

36 Man schātzt die Zahl der aus den Ortschaften im Süden Luxemburgs, d.h. in Vorgebiet der Maginot-Linie, nach Zentralfrankreich evakuierten Menschen zwischen 44760 und 52 000, die der ins nördliche Luxemburg auf rd. 49 000, vgl. J. Meyers, Geschichte Luxemburgs, Luxemburg 1969, S. 145 und P. J. Müller, Tatsachen aus der Geschichte Luxemburgs, Luxemburg 1968.

37 Vgl. Serebreniks Angaben in: Eichmannprozeß Dok. 297; femer Charles et Graziella Lehrmann, La Communauté juive du Luxembourg dans la passé et dans le présent, Luxembourg 1963, S. B5; Bosseler/ Steichen, Livre d'Or, S. 569; Cerf, Longtemps, S. 28.

38 Cerf erwähnt mehrere Konvois, die mit Hilfe der deutschen Wehrmacht, insbesondere des Chefs der Passierscheinstelle, Baron von Hoiningen-Huene, mit Ziel Spanien und Portugal organisiert werden konnten, Cerf, Longtemps, S. 35. Vgl. auch Henri Koch-Kent, Sie boten Trotz. Luxemburg im Freiheitskampf 1939-1945, Luxemburg 1974, S. 88. Folgt man der Angabe, die Serebrenik im April 1941 (vgl. Anm. 46) Eichmann gegenüber machte, so haben zwischen Mai 1940 und April 1941 ca. 3000 aus Luxemburg emigrieren können, er selber hat laut Eichmannprozeß, Dok. 297, 2500 Juden zur Auswanderung verholfen. 
die nach Erlaß der Verordnung vom 5. September 1940 als Juden im Sinn der nationalsozialistischen Definition bezeichnet wurden. ${ }^{39}$ Trotz dieser verschiedenen Unsicherheitsfaktoren abet kann man davon ausgehen, daß Mitte September zwischen 1700 und 2000 Juden in Luxemburg lebten - 65\% in der Hauptstadt und $35 \%$ in kleineren Orten. 40

Der zweite Exodus der Luxemburger Juden begann im Herbst 1940 nach einer ihnen von Gauleiter Simon und der Gestapo angedrohten Massenausweisung. ${ }^{41}$ Wenn es nicht gelānge, so wurde dem Großrabbiner und dem Konsistorium am 12. September bedeutet, innerhalb von zwei Wochen die Auswanderung der gesamten jüdischen Bevōlkerung zu organisieren, so würde sie nach Ablauf dieser Frist an Jom Kippur, dem Versōhnungstag und hōchsten jüdischen Feiertag, in Sammeltransporten abgeschoben. Ob die an den Präsidenten der Verwaltungskommission, Albert Wehrer, gerichtete beschwōrende Denkschrift ${ }^{42}$, mit der das Konsistorium um eine Demarche bei den deutschen Behörden bat, oder eine Intervention des amerikanischen Geschäftsträgers, George $\mathrm{P}$. Waller, diese Maßnahme verhindert hat bzw. ob diese Drohung nicht vielmehr das Mittel zum Zweck darstellte, muß unbeantwortet bleiben.

Die Ausweisung also ist unterblieben, die Auswanderung aber wurde, zweifellos unter dem Eindruck des eben noch abgewendeten Schicksals, forciert. Zwischen August 1940 und Oktober 1941 konnten 387 Juden nach Portugal und 50 nach Spanien auswandern, und vom 22. Oktober 1940 bis zum 21. Januar 1941 haben in 14 kleineren oder grōßeren Transporten 653 Juden das Großherzogtum in Richtung (meist unbesetztes) Frankreich und 49 in Richtung Belgien mit dem weiteren Ziel Ubersee verlassen. ${ }^{43}$ Das endgültige Emigrationsland haben nur wenige erreicht, viele - schätzungsweise $70 \% 44$ - kamen nicht weiter als bis Frankreich und wurden schließlich mit den

39 Im Oktober 1944 gab es noch acht sog. Halbjuden in Luxemburg, vgl. Cerf, Longtemps, S. 125; entsprechende Angaben für frühere Jahre sind nicht bekannt.

40 In einer Denkschrift des Konsistoriums (abgedruckt bei Cerf, Longtemps, S. 142-146) wird die Zahl 2000 genannt, die, berücksichtigt man die von Serebrenik im April 1941 genannte Zahl von 947 (vgl. Anm. 46) in erwa den Tatsachen entspricht Zihlt man zu diesen 947 die 702 zwischen Oktober 1940 und Januar 1941 nach Frankreich und Belgien sowie die 437 nach Portugal und Spanien evakuienen Juden (vgl. Anm. 43) hinzu, so kommt man aul insgesamt 2086 Personen.

41 Dies und des folgende, soweit nicht anders angegeben, nach Cerf, Longtemps, S. $42 \mathrm{ff}$. und Cert, L'Etoile Juive, S. 53 ft.

42 Vgl. Anm. 40.

43 Quelle für diese Zahl ist ein im Livre d'Or enthaltenes Tableau d'Honneur (S. 574-594), ein namentliches Verzeichnis der zwischen 22.10.1940 und 6.4.1943 aus Luxemburg evakuierten bzw. deportierten Juden. Cerf, Longtemps, S. 50, hat diese Angaben hinsichtlich Zahl, Stärke, Ziel und Termin der Transporte nach Frankreich und Belgien übernommen; die von ihm errechnete Gesamtzahl von 619 (statt 697) in diesen 14 Konvois weggeschafften Juden ist entweder auf einen Additions- oder Druckfehler zurückzuführen. In Cerfs neuester Veröffentlichung aus dem Jahre 1986 umfaßt der Konvoi, der am 21.1.1941 Luxemburg in Richtung unbesetztes Frankreich verlief, fünf Personen mehr, daher ergibt sich nun eine Gesamtzahl von 702 evakuierten Juden, Cerf, L'Etoile Juive, S. 69.

44 Cerf, Longtemps, S. 128. Auf welcher Grundlage diese Schätzung beruht, ist nicht bekannt. Des von Serge Klarsfeld zusammengestellte „Mémorial de la Déportation des Juifs de France“, Paris 1978, kann, da nicht alle Deportationslisten auch die Nationalitāt der jeweils Verschleppten ausweisen, nur zur Klärung einzelner Luxemburger Juden, nicht aber als Quelle für die Zahl der aus Frankreich in die Vernichtungslager deportierten Juden aus dem Grotherzogtum dienen. Insgesamt konnten 184 luxemburgische Juden, die aus Frankreich und 31, die aus Belgien deportiert wurden, namentlich ermittelt werden. Von diesen insgesamt 215 Personen haben 16 überlebt, Cerf, L'Etoile Juive, S. 144 und 208 f. 
dort Ende März 1942 einsetzenden Transporten in die Lager des Ostens deportiert. ${ }^{45}$

Wāhrend einer Besprechung im Reichssicherheitshauptamt (RSHA) am 24. April 1941 bei Eichmann über Möglichkeiten, die Auswanderung zu beschleunigen ${ }^{46}$, gab Großrabbiner Serebrenik die Zahl der noch im Großherzogtum lebenden Juden mit 947 (413 Mānner und 534 Frauen) an, von denen 350 auswanderungswillig und zum Teil (100 Personen) schon im Besitz der notwendigen Papiere seien. In den folgenden knapp sechs Monaten aber, d.h. zwischen Ende April und Mitte Oktober 1941, als die Auswanderung auf Anweisung Himmlers gestoppt wurde, konnten nur noch etwa 250 Juden, darunter am 26. Mai 1941 auch der GroBrabbiner, emigrieren. 47 Insgesamt hatten seit August 1940 etwa 1450 Juden Luxemburg verlassen. 48

Im August 1941 begann man mit der Konzentrierung vor allem alter, kranker und nach Luxemburg geflohener Juden in einer Art Ghetto im Kloster Fünfbrunnen (Cingfontaine) im Norden Luxemburgs, nahe Uflingen (Troisvierges), das bereits im März 1941 von der Gestapo weitgehend geräumt worden war. Die ersten 25 Personen trafen am 11. August in dem strategisch günstig an der in nord-südlicher Richtung verlaufenden Bahnlinie gelegenen Kloster ein. Von dieser Sammelstelle aus konnten die Juden gruppenweise in die Mitte Oktober beginnenden Transporte nach Polen, die in Trier den aus dem Reichsgebiet abgehenden angeschlossen wurden, eingereiht werden.

Noch bevor der "Arbeitseinsatz im Osten " auch für die luxemburgischen Juden Wirklichkeit wurde, waren bereits 94 Juden sechs Wochen lang zur Zwangsarbeit in einem Steinbruch an der Mosel und beim Straßenbau in der Nähe von Wittlich eingesetzt worden. ${ }^{49}$ Diese Maßnahme bewahrte sie davor, schon mit dem ersten und größten Transport am 16. Oktober 1941 von Luxemburg nach Lodz (Litzmannstadt) ${ }^{50}$ de-

45 $\mathrm{Zu}$ den bis zum 18.7.1943 aus dem altbesetzeen“ Frankreich deporienten etwa $\$ 2000$ Juden gehörten, entsprechend einer vom Mär 1942 datierten Entscheidung (vgl. Schreiben des SS-Hauptsturmführers Theodor Dannecker vom 17.3.1942 an den Militäbefehlshaber Frankreich, Eichmannprozeß, Dok. 587), auch jene mit luxemburgischer Staatsangehörigkeit (vgl. Vermerk SS-Obersturmführer Heinz Röthke, BdS Paris, vom 21.7.1943, Eichmannprozeß, Dok. 664).

46 Uber die Besprechung im RSHA hat Serebrenik am 21.7.1960 während des Eichmann-Prozesses in Jerusalem einen - mit Ausnahme einiger in deutsch wiedergegebener Zitate von Bemerkungen Eichmanns - nur in hebräisch vorliegenden Bericht gegeben, der in der 37. Sitzung des Gerichts, am 11. Mai 1961 als Beweisdokument 297 in den Prozeß eingeführt worden ist (vgl. auch Anm. 40). Die Angabe des Großrabbiners "März 1941" beruht vermutlich auf einem Erinnerungsfehler, sowohl bei Cerf wie bei Evy wird April 1941 angegeben, vgl. Cerf, L'Etoile, S. 86-89. Cerf, Longtemps, S. 45-50, referiert wesentliche Teile der Unterredung, über die Serebrenik offenbar schon vier Wochen vorher, am 27.6.1960, und zwar noch ausführlicher, berichtet hat.

47 Cerf, Longtemps, S. So; Evy, Fünfbrunnen, Nr. 23, S. 42.

48 Cerf, L' Etoile S. 60 und 177

49 Ebenda, S. 105 und Cerf, Longtemps, S. 74.

50 Die Ankunft dieses Transportes, dem noch 178 Juden auf der Fahrt durch Deutschland angeschlossen wurden, am 18.10.1941 in Lodz ist dokumentarisch zweifach überliefert: Einmal durch ein Schreiben des „Ältesten der Juden in Litzmannstadt ${ }^{\mu}$, Chaim Rumkowski, vom 13.11.1941 an die Luxemburger Kultusgemeinde (Faksimile bei Cerf, Longtemps, S. 101) und durch den "Erfahrungsbericht betr. Einweisung von 20000 Juden und 5000 Zigeuner in das Ghetto Litzmannstadt “ des Hauptmanns der Schutzpolizei, Künzel mit gleichem Datum (vgl. Dokumenty i Materialy, Tom III, Warszawa 1946, S. 203 ff.). Insgesamt 54490 Insassen des Lodzer Ghettos - darunter 10993 der 2 wischen dem 16. Oktober und dem 4. November 1941 dort eingewiesenen fast 20000 Juden aus dem Atreich, Osterreich und dem Protektorat - sind in 66 Transporten zwischen Mitte Januar und Mitte Mai 1942 nach Chelmno gebracht worden (vgl. FaschismusGetto-Massenmord, hrsg. vom Jüdischen Historischen Institut Warschau, Berlin $1961^{2}$, S. 285 f. und Adalbert Rückerl, NS-Vernichtungslager im Spiegel deutscher Strafprozesse. Belzec, Sobibor, Treblinka, Chelmno, München 1977, S. 257 ff.). 
portiert zu werden, einen Tag nachdem der letzte Auswandererkonvoi mit etwa 120 Juden Luxemburg in Richtung Spanien verlassen hatte. Die meisten der 331 Verschleppten sind - sofern sie die ersten Wochen des Ghettoaufenthaltes in Lodz überhaupt überlebt haben - mit an Sicherheit grenzender Wahrscheinlichkeit mit den Mitte Januar 1942 einsetzenden Transporten in das Vernichtungslager Chelmno deportiert worden.

Nachdem der erste Transport das Großherzogtum verlassen hatte, war die Zahl der Juden auf 362 zurückgegangen. ${ }^{51}$ Ein halbes Jahr später setzte man die Transporte fort und deportierte in sechs weiteren Aktionen bis Mitte Juni 1943 insgesamt 352 Juden, davon wurden 27 in das als Durchgangslager für Belzec bekannte Izbica eingeliefert, 32 kamen nach Auschwitz und 286 nach Theresienstadt. Weitere sieben wurden mit unbekanntem Ziel deportiert.52 Verschont blieben weder Geisteskranke noch Kinder und Greise. 53 Fünf Patienten der Heil- und Pflegeanstalt Ettelbrück kamen mit dem Transport vom 28. Juli 1942 nach Theresienstadt, sieben - vom Anstaltsarzt für transportunfähig erklärt - am 17. Juni 1943 direkt nach Auschwitz. Nach Kriegsende zählte man 43 Úberlebende, das waren weniger als 6,5\% der 683 Deportierten. ${ }^{54}$ Die Zahl derer, die in Luxemburg überlebt haben, dürte etwa 20 bis 30 betragen haben.

Außer den unmittelbar aus Luxemburg nach dem Osten deportierten und dort umgekommenen Juden hat auch die meisten der nach Frankreich und Belgien ca. 2950 geflohenen bzw. evakuierten Juden das gleiche Schicksal getroffen. 600 konnten sich durch Emigration ins neutrale bzw. befreundete Ausland retten. Etwa 1850 überlebten im Untergrund oder versteckt in Frankreich und Belgien. Von dort deportiert wurden

51 Die Zahl von 362 noch in Luxemburg lebenden Juden (216 davon alter als 60 Jahre) meldete das Konsistorium am 14.11.1941 der Gestapo, vgl. Cerf, Longtemps, S. 85 f.

52 Alfred Oppenheimer, Mitte April 1942 von den deutschen Behörden zum sog. Judeniltesten bestimmt, machte in zwei Aussagen für den Eichmann-ProzeB (am 6.12.1960 in Luxemburg. vgl. Cerf, Longtemps, S. 216-220, und am 7.6.1961 in Jerusalem, vgl. Eichmannprozeß, Dok. 1075) hinsichtlich der Stärke und der Abgangsdaten der Transporte nach Polen und Theresienstadt Angaben, die von jenen, die im Livre d'Or enthalten sind, geringfügig abweichen. Oppenheimer, im Juli 1943 nach Theresienstadt und von dort Anfang Oktober 1944 nach Auschwitz deportiert, zähle aufgrund seiner Unterlagen 674 Deportierte (36 Zurückgekehrte) in acht Transporten bis zum 28.9.1943. Die sieben Listen im Livre d'Or schließen mit dem Transport vom 6.4.1943 ab und führen insgesamt 728 Deportierte namentlich, einige allendings doppelt auf. Cerf, Longtemps, S. 198-213 kommt auf der Grundlage der aktualisierten Listen aus dem Livre d'Or zu einer Gesamtzahl von 670 Deportierten (42 Uberiebende), so daß die Differenz zwischen den Angaben von Oppenheimer und denen von Cerf, Longtemps (der zwei - It. Oppenheimer - noch im September 1943 nach Theresienstadt Deportierte nicht berücksichtigt) minimal ist. Cerf, L'Etoile, S. 179-200 weist in einer abemals korrigienten namentlichen Aufstellung 683 Deportierte (43 Uberiebende) nach. Hier werden nach Lodz 331 Personen im Gegensatz 2u Cerf, Longtemps, 334 deportiert (in Cerf, Longtemps, sind es insgesamt sieben Personen, die nicht in Cerf, L'Etoile, auftauchen, dafür sind dort vier Namen angegeben, die nicht in Cerf, Longtemps erscheinen, so daß bei Cerf, L'Etoile insgesamt drei Personen weniger zu verzeichnen sind). Bei Cerf, L'Etoile, sind zusätzlich zu Cerfs vorhergehender Veröffentlichung Michael Levy, Berthe Levy, geb. Bermann und Roger Levy nach Izbica deportiert worden. Der Transport vom 26. Juli 1942 nach Theresienstadt enthält in Certs Publikation aus dem Jahre 1986 zusätzlich die Namen von Mathilde Cahen, Ernst Geiershōfer und Julie Hartoch-Wolf. Bei dem Transport vom 28. Juli 1942 dagegen erscheint Martin Cohn in der Veröffentlichung von 1986 nicht mehr, dafür aber: Alexander Herlinger, Berha Herlinger, geb. Hermann, Hugo Neumann und Selma Neumann, geb. Dalberg. Zusatzlich gibe Cert, L'Etoile, S. 200, noch sieben Deportierte mit verschiedenen unbekannten Zielen an. Bezüglich der Zahl der Zurückgekehrten dürtten die Angaben bei Cerf, der sich auf in Luxemburg verwahrtes Material stützen konnte, zutreffen. Zum Schicksal der nach Theresienstadt und von dort weiter Deportierten vgl. H.G. Adler, Theresienstadt. Antlitz einer Zwangsgemeinschaft, Tübingen $1960^{2}$, S. $45-60$.

53 Cerf, Longtemps, S. $107 \mathrm{f}$. und $209 \mathrm{ff}$.

$34 \mathrm{Vgl}$. Anm 52. 
zwischen 370 und 400 (16 bis 20 Uberlebende), wovon 215 namentlich bekannt sind und in den von Serge Klarsfeld\$s bzw. Serge Klarsfeld und Maxime Steinberg ${ }^{56}$ zusammengestellten Listen der Deportationszüge erscheinen. Weitere zwölf wurden in Konzentrationslager nach Deutschland bzw. Österreich gebracht und starben dort. Man kann also davon ausgehen, daß von jenen Luxemburger Juden, die die Deutschen aus Frankreich oder Belgien deportierten, etwa 380 umgekommen sind. 57 Opfer des nationalsozialistischen Regimes sind aber auch jene Juden geworden, die infolge der minimalen Existenzbedingungen den $\mathbf{Z}$ wangsaufenthalt in Fünfbrunnen nicht überlebt haben 58 sowie jene, die aus Verzweiflung über die ihnen bevorstehende Deportation den Freitod gewählt haben; ihre Zahl hat z. B. bei dem Transport nach Lodz acht betragen. 59

Damit ergibt sich folgende geschātzte Gesamtbilanz der jüdischen Menschenverluste in Luxemburg: Von den ca. 3500 bis 3700 am 10. Mai 1940 im Großherzogtum lebenden Juden sind mindestens 1200 , d.h. rd. $35 \%$, der nationalsozialistischen Verfolgung zum Opfer gefallen. (Yad Vashem beziffert die Zahl mit 1950, Hilberg schātzt sie dagegen auf unter 1000.) Davon wurden allerdings nur etwa 720 Juden aus Luxemburg selbst deportiert, bzw. sind dort im Rahmen der Verfolgung verstorben. Die übrigen wurden über Frankreich und Belgien in die Vernichtungslager des Ostens verschickt. Wieviele von den 1200 Opfern luxemburgischer Nationalitāt waren, ist nicht zu klären. Auf jeden Fall aber hatten die in den französischen und belgischen Deportationslisten aufgeführten namentlich bekannten 215 Juden die luxemburgische Staatsangehörigkeit.

5s Vgl. Klarsfeld, Le Mémorial.

56 Vgl. Serge Klarsfeld/Maxime Steinberg, Mémorial de la Déporation des Juifs de Belgique, Bruxelles 1982.

57 Cerf, L'Etoile, S. 177.

58 Dazu insbesondere Cerf, Longtemps, S. 86-94; in Bosseler/Steichen, Livre d'Or, S. 571 wird die Zahl auf 20 geschätzt Evy (Fünfbrunnen. Nr. 23, S. 43) nennt, unter Berufung auf die Angabe eines Klosterbruders, 15 in Cingfontaine Verstorbene.

59 Cerf, Longtemps, S. 80 